\title{
Le Lièvre et les Grenouilles de Jean de La Fontaine
}

Traduction et note à la traduction

\section{Luca Pietromarchi}

\section{(2) OpenEdition}

Journals

Édition électronique

URL : http://journals.openedition.org/rief/283

DOI : 10.4000/rief.283

ISSN : 2240-7456

Éditeur

Seminario di filologia francese

Référence électronique

Luca Pietromarchi, «Le Lièvre et les Grenouilles de Jean de La Fontaine », Revue italienne d'études françaises [En ligne], $3 \mid 2013$, mis en ligne le 15 décembre 2013, consulté le 22 septembre 2020. URL: http://journals.openedition.org/rief/283 ; DOI : https://doi.org/10.4000/rief.283

Ce document a été généré automatiquement le 22 septembre 2020.

\section{(c) (7) $\odot$}

Les contenus de la RIEF sont mis à disposition selon les termes de la Licence Creative Commons Attribution - Pas d'Utilisation Commerciale - Pas de Modification 4.0 International. 


\section{Le Lièvre et les Grenouilles de Jean de La Fontaine}

Traduction et note à la traduction

Luca Pietromarchi

\section{RÉFÉRENCE}

Le Lièvre et les Grenouilles, Fables (1668), II, 14, dans Euvres complètes, éd. J.-P. Collinet, Paris, Gallimard, «Bibliothèque de La Pléiade », vol. I, 1991, p. 89-90

\section{Version originale}

Un Lièvre en son gîte songeait

(Car que faire en un gîte, à moins que l'on ne songe ?) ;

Dans un profond ennui ce Lièvre se plongeait :

Cet animal est triste, et la crainte le ronge.

« Les gens de naturel peureux 5

Sont, disait-il, bien malheureux ;

Ils ne sauraient manger morceau qui leur profite.

Jamais un plaisir pur, toujours assauts divers.

Voilà comme je vis : cette crainte maudite

M'empêche de dormir, sinon les yeux ouverts. 10

Corrigez-vous, dira quelque sage cervelle.

Et la peur se corrige-t-elle?

Je crois même qu'en bonne foi

Les hommes ont peur comme moi ».

Ainsi raisonnait notre Lièvre 15

Et cependant faisait le guet.

Il était douteux, inquiet :

Un souffle, une ombre, un rien, tout lui donnait la fièvre. 
Le mélancolique animal

En rêvant à cette matière, 20

Entend un léger bruit : ce lui fut un signal

Pour s'enfuir devers sa tanière.

Il s'en alla passer sur le bord d'un étang.

Grenouilles aussitôt de sauter dans les ondes,

Grenouilles de rentrer en leurs grottes profondes. 25

«Oh! dit-il, j'en fais faire autant

Qu'on m'en fait faire ! Ma présence

Effraie aussi les gens, je mets l'alarme au camp!

Et d'où me vient cette vaillance?

Comment! des animaux qui tremblent devant moi ! 30

Je suis donc un foudre de guerre!

Il n'est, je le vois bien, si poltron sur la terre

Qui ne puisse trouver un plus poltron que soi ».

\section{Traduction italienne}

Meditava una Lepre nella tana

(che fare in una tana, se non meditare ?) ;

nella noia più profonda la Lepre era immersa, quest'animale triste, che la paura divora.

«Le persone paurose di natura 5

sono, diceva, davvero infelici ;

non un solo boccone da assaporar con gusto.

Mai un piacere puro, sempre e solo a spizzico.

Mi tocca viver così : questa dannata paura

m'impedisce di dormire, se non gli occhi aperti. 10

Curatevi, mi dirà il primo sapientone.

Ma si guarisce dalla paura?

A ben vedere direi anzi

che gli uomini han paura come me ".

Così ragionava la Lepre 15

mentre stava di vedetta.

Era ansiosa, sempre inquieta :

un soffio, un'ombra, un niente, tutto la spaventava.

Il malinconico animale,

mentre a queste cose pensava, 20

sente un leggero rumore : fu quello il segnale

che lo fece fuggir verso la tana.

Passò quindi davanti alla riva di uno stagno.

Rane che subito saltano nell'onda,

rane che scappano in grotte profonde. 25

«Caspita! metto tanta paura

quanta ne mettono a me ! Eccomi,

e tutti si spaventano, tutti gridano allarme!

Chi mi ha dato una simil prestanza?

Ma come ! animali tremanti al mio cospetto ! 30 
Son dunque un fulmine di guerra!

Non c'è, l'ho ben capito, fifone in terra

che trovar non possa uno ancor più fifone ».

\section{Note à la traduction}

1 Dans une lettre à sa femme, le 5 septembre 1663, La Fontaine, en voyage vers le Limousin, lui décrit en ces termes le château de Blois :

Il y a en face un corps de logis à la moderne, que feu Monsieur a fait commencer : toutes ces trois pièces ne font, Dieu merci, nulle symétrie, et n'ont rapport ni convenance l'une avec l'autre ; l'architecte a évité cela autant qu'il a pu. Ce qu'a fait faire François I, à le regarder du dehors me contenta plus que tout le reste : il y a force petites galeries, petites fenêtres, petits balcons, petits ornements, sans régularité et sans ordre ; cela fait quelque chose de grand qui plaît assez. ${ }^{1}$

2 Goût pour le renouvellement d'un corps ancien; horreur ("Dieu merci ») de la symétrie; plaisir suscité par le principe de discontinuité, par le choc que produit la rupture de la ligne et de toute esthétique fondée sur la régularité, rabaissée à la faveur d'une incohérence, voire d'une inconvenance, destinée à susciter la surprise et l'étonnement. N'est-il pas loisible de reconnaître en cette description de Blois les éléments d'une esthétique qui, au-delà de son objet architectural, investit la poétique de La Fontaine, à savoir le principe de composition des Fables? Le chiasme de la dernière phrase, en soulignant l'effet de grandeur et de plaisir suscité par l'irrégularité et le désordre du bâtiment, fait en effet écho à toutes les propositions de La Fontaine concernant son art de la composition poétique, dont « le secret de plaire ne consiste pas toujours en l'ajustement, ni même en la régularité $»^{2}$. Le poète, ainsi que l'architecte et son roi, « a évité cela autant qu'il a pu ».

3 Il suffira en effet de regarder une fable de La Fontaine pour voir surgir la silhouette du château de Blois, à tel point la variété des mètres employés y est abondante, avec son alternance d'alexandrins et d'octosyllabes ou de décasyllabes qui ne cesse de briser et de relancer le rythme du texte. De même, le jeu des rimes y est fort souvent irrégulier, toujours soumis à l'intention de mimer les soubresauts, les étonnements, les effets de surprise que La Fontaine ne cesse d'aménager afin de vivifier l'apologue et de le dégager de sa traditionnelle gaine moralisante.

4 La Fable II, 14, Le Lièvre et les Grenouilles, est, de ce point de vue, exemplaire. Toutes les ressources dynamisantes de la versification concourent à mettre à nu, dans la description de ce grand mélancolique qu'est le Lièvre, sa nature névrotique. Et c'est avec un entrain véritablement névrotique que la Fontaine accumule les entorses qu'il fait subir à la prosodie classique pour mimer l'inquiète mobilité du lièvre, cet effrayé qui, dans un renversement presque pascalien, se découvre effrayant. Les alternances métriques, les répétitions qui foisonnent dans les deux premiers vers, l'élision du sujet (v. 16), l'enjambement (v. 27), l'emploi de l'infinitif historique sans conjonction (v. 24-25) : ce sont autant d'éléments prosodiques qui concourent à produire un rythme qui mime la respiration haletante de l'animal en fuite. C'est à cet aspect que la traduction se doit d'être le moins infidèle, quitte à bousculer la mesure du vers italien traditionnel, surtout du vers court. Celui-ci est, dans cette fable, l'octosyllabe, auquel nous avons fait correspondre une gamme métrique qui va de l'octosyllabe au vers de neuf syllabes, et jusqu'à l'hendécasyllabe, transférant à l'ensemble constitué par les octosyllabes le principe d'alternance dont le poète s'autorise en mêlant vers longs et 
courts. Lexicalement, nous avons recherché la plus grande adhérence à l'original afin de restituer le relief propre à la narration de cette aventure champêtre, où chaque détail éveille, non seulement l'inquiétude du lièvre, mais l'attention du lecteur. De ce point de vue, le prix de la rime ou du rigoureux respect de la versification italienne, avec ses inévitables élisions lexicales et ses inversions anoblissantes, nous a semblé exorbitant par rapport à la souplesse de la syntaxe originale. De même, lorsque la traduction littérale, comme dans le cas de "poltron» dans le distique final, offrait un terme équivalent en italien mais seulement dans son acception ancienne, nous avons préféré (au contraire de De Marchi, 1885 : « poltrone », et de Valeri, 1952 : «vile ») un terme plus moderne : «fifone », qui nous a semblé, ne serait-ce qu'à l'égard du sujet, plus courant...

5 Ne jamais «négliger le plaisir du cœur pour travailler à la satisfaction de l'oreille $»^{3}$ : cette justification que donnait La Fontaine pour excuser les licences métriques de ses contes, n'y aurait-il pas lieu de l'entendre comme une admonestation à l'égard de ses futurs traducteurs?

\section{NOTES}

1. La Fontaine, Relation d'un voyage de Paris en Limousin, in CEuvres diverses, éd. P. Clarac, Paris, Gallimard, «Bibliothèque de la Pléiade », 1958, p. 544, cit. dans J. D. Biard, Le Style des fables de La Fontaine, Paris, Nizet, 1969, p. 40.

2. La Fontaine, Deuxième partie des Contes et Nouvelles en vers, «Préface ", dans Cuvres diverses, cit., p. 603.

3. Ibidem.

\section{INDEX}

Mots-clés : traduction, vers classique, Fables, La Fontaine (Jean de), hétérométrie 